\title{
Correction to: Effects of eculizumab treatment on quality of life in patients with paroxysmal nocturnal hemoglobinuria in Japan
}

\author{
Yasutaka Ueda ${ }^{1,2} \cdot$ Naoshi Obara $^{2,3} \cdot$ Yuji Yonemura ${ }^{2,4} \cdot$ Hideyoshi Noji $^{2,5} \cdot$ Masayoshi Masuko $^{6}$. \\ Yoshinobu Seki ${ }^{7,8} \cdot$ Katsuya Wada $^{9} \cdot$ Takahisa Matsuda $^{10}$ - Hirozumi Akiyama ${ }^{10} \cdot$ Takayuki Ikezoe $^{2,5}$.

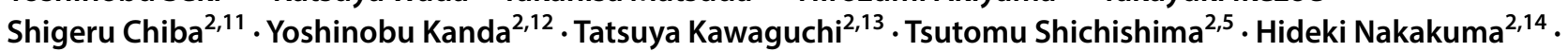 \\ Shinichiro Okamoto ${ }^{2,15} \cdot$ Jun-ichi Nishimura ${ }^{1,2} \cdot$ Yuzuru Kanakura ${ }^{1,2} \cdot$ Haruhiko Ninomiya $^{2,16}$ (D)
}

Published online: 6 July 2018

(c) The Japanese Society of Hematology 2018

\section{Correction to: \\ International Journal of Hematology (2018) 107:656-665 \\ https://doi.org/10.1007/s12185-018-2409-3}

In the original publication of this article, Tables 2, 3 and 4 were published incorrectly. The corrected Tables 2, 3 and 4 are given in the following pages.
The original article can be found online at https://doi.org/10.1007/ s12185-018-2409-3.

\section{Haruhiko Ninomiya}

ninomiya.haruhiko.ft@u.tsukuba.ac.jp

1 Department of Hematology and Oncology, Osaka University Graduate School of Medicine, Suita, Osaka, Japan

2 Japan PNH Study Group, Tokyo, Japan

3 Department of Hematology, University of Tsukuba, Tsukuba, Japan

4 Department of Transfusion Medicine and Cell Therapy, Kumamoto University Hospital, Kumamoto, Japan

5 Department of Hematology, Fukushima Medical University, Fukushima, Japan

6 Department of Stem Cell Transplantation, Niigata University Hospital, Niigata, Japan

7 Department of Hematology, Niigata Prefectural Shibata Hospital, Shibata, Japan

8 Department of Hematology, Uonuma Institute of Community Medicine, Niigata University Medical and Dental Hospital, Minami-Uonuma, Japan
9 Department of Hematology, Matsushita Memorial Hospital, Moriguchi, Japan

10 Alexion Pharma G.K, Tokyo, Japan

11 Department of Hematology, Faculty of Medicine, University of Tsukuba, Tsukuba, Japan

12 Division of Hematology, Jichi Medical University, Shimotsuke, Japan

13 Departments of Hematology and Infectious Diseases, Kumamoto University, Kumamoto, Japan

14 Kagoshima Tokusyukai Hospital, Kagoshima, Japan

15 Division of Hematology, Department of Medicine, Keio University School of Medicine, Tokyo, Japan

16 Department of Medical Sciences, Faculty of Medicine, University of Tsukuba, Tsukuba, Japan 
Table 2 Disease characteristics at baseline

Table 3 Changes in laboratory data from baseline to 1 -year

\begin{tabular}{lllll}
\hline & Post-marketing & QOL & BMF $(+)$ & BMF (-) \\
\hline LDH (U/L) & & & & \\
$n$ & 477 & 51 & 30 & 21 \\
Mean (SD) & $1478.3(776.3)$ & $1435.3(922.6)$ & $1161.8(591.4)$ & $1826.0(1161.2)$ \\
Median (min, max) & $1340.0(157,6082)$ & $1131.0(447,6082)$ & $1049.0(447,2870)$ & $1638.0(618,6082)$ \\
Hb (g/dL) & & & 31 & 21 \\
$n$ & 480 & 52 & $8.17(2.19)$ & $8.29(1.47)$ \\
Mean (SD) & $8.04(1.70)$ & $8.22(1.92)$ & $8.20(4.5,15.0)$ & $8.20(5.7,11.5)$ \\
Median (min, max) & $7.90(3.8,15.0)$ & $8.20(4.5,15.0)$ & & 21 \\
PLT (× 10 $3 / \mu L)$ & & & 30 & $166.4(66.6)$ \\
$n$ & 451 & 51 & $103.0(59.3)$ & $172.0(60,281)$ \\
Mean (SD) & $133.3(80.6)$ & $129.1(69.3)$ & $93.0(12,228)$ & 12 \\
Median (min, max) & $126.0(4,574)$ & $128.0(12,281)$ & & $37.28(23.81)$ \\
PNH clone size (\%) & & & 14 & $36.70(1.7,83.5)$ \\
$n$ & 241 & 26 & $19.20(10.2,97.0)$ & \\
Mean (SD) & $42.18(23.90)$ & $34.94(26.66)$ & $32.93(29.62)$ & \\
Median (min, max) & $40.00(0.0,99.0)$ & $23.35(1.7,97.0)$ & & \\
\hline
\end{tabular}

PNH clone size (\%) was evaluated by erythrocytes

\begin{tabular}{|c|c|c|c|}
\hline & Baseline & 1 year & Paired $t$ test \\
\hline \multicolumn{4}{|l|}{$\mathrm{LDH}(\mathrm{U} / \mathrm{L})(n=51)$} \\
\hline Mean (SD) & $1435.3(922.6)$ & $292.2(170.7)$ & \multirow[t]{2}{*}{$P<0.01$} \\
\hline Median (min, $\max )$ & $1131.0(447,6082)$ & $242.0(148,1045)$ & \\
\hline \multicolumn{4}{|l|}{$\mathrm{Hb}(\mathrm{g} / \mathrm{dL})(n=52)$} \\
\hline Mean (SD) & $8.22(1.92)$ & $9.37(1.97)$ & \multirow[t]{2}{*}{$P<0.01$} \\
\hline Median (min, max) & $8.20(4.5,15.0)$ & $9.35(5.1,14.3)$ & \\
\hline \multicolumn{4}{|c|}{$\operatorname{PLT}\left(\times 10^{3} / \mu \mathrm{L}\right)(n=51)$} \\
\hline Mean (SD) & $129.1(69.3)$ & $133.8(64.8)$ & \multirow[t]{2}{*}{ N.S. } \\
\hline Median (min, max) & $128.0(12,281)$ & $137.0(11,306)$ & \\
\hline \multicolumn{4}{|c|}{$\mathrm{eGFR}\left(\mathrm{mL} / \mathrm{min} / 1.73 \mathrm{~m}^{2}\right)(n=29)$} \\
\hline Mean (SD) & $73.75(37.87)$ & $75.57(38.47)$ & \multirow[t]{2}{*}{ N.S. } \\
\hline Median (min, max) & $58.60(27.0,161.3)$ & $64.80(25.2,171.1)$ & \\
\hline \multicolumn{4}{|c|}{ PNH clone size $(\%)(n=5)$} \\
\hline Mean (SD) & $28.88(30.81)$ & $33.60(16.41)$ & \multirow[t]{2}{*}{ N.S. } \\
\hline Median (min, $\max$ ) & $18.80(11.0,83.5)$ & $23.50(19.3,52.0)$ & \\
\hline
\end{tabular}

PNH clone size (\%) was evaluated by erythrocytes 
Table 4 Canonical correlation analysis between changes in laboratory data and changes in QOL scores

Canonical correlation coefficient

\begin{tabular}{lr}
\hline FACIT-fatigue & 0.74 \\
EORTC-QLQ-C30 & \\
Global health status/QoL & 0.69 \\
Physical functioning & 0.72 \\
Role functioning & 0.64 \\
Emotional functioning & 0.38 \\
Cognitive functioning & 0.20 \\
Social functioning & 0.59 \\
Fatigue & -0.87 \\
Nausea and vomiting & -0.44 \\
Pain & -0.27 \\
Dyspnea & -0.70 \\
Insomnia & -0.58 \\
Appetite loss & -0.55 \\
Constipation & -0.12 \\
Diarrhea & -0.16 \\
Financial difficulties & -0.51 \\
Laboratory value & \\
LDH & -0.91 \\
Hb & 0.76 \\
PLT & 0.03
\end{tabular}

Statistics

Canonical $R$

0.8393

$F$ value

1.9166

$P$ value

0.0042 